\title{
The ultrasound image patterns at the early stage after birth can predict health neonates - -a nested case-control study
}

\author{
Guannan $\mathrm{Xi}^{1}$, Jiale Dai ${ }^{1}$, Wang Xuefeng ${ }^{1}$, Fei Luo ${ }^{1}$, Chengqiu Lu ${ }^{1}$, Yun Yang ${ }^{1}$, and Jimei \\ Wang $^{1}$
}

${ }^{1}$ Obstetrics and Gynecology Hospital of Fudan University

June 1, 2020

\begin{abstract}
Abstract Purpose To distinguish healthy infants from potential patients right after birth using lungultrasound(LUS). Design, Setting and Patients This is a nested case-control study containing 22 lung diseases patients and 473 healthy infants from a total of 504 successive infants. They are admitted to Obstetrics \& Gynecology Hospital of Fudan University, Shanghai, China, from 1st January 2020 to 1st April 2020. A newly designed scanning protocol is used to capture LUS images. Sensitivity, specificity, PPV and NPV for predicting healthy infants and patients were calculated individually. The transition process image patterns and thier variation were shown. The relationship between clinic signs and the high-risk image patterns was calculated by Kendall's tau-b test. Measurements and main results LUS images were captured and its preditive value has been caculated. Four low-risk patterns almost only can be seen in healthy infants(specificity=86.4\%, PPV=99.0\%) whereas four high-risk patterns can be seen both in healthy infants and patients(specificity $=62.4 \%, \mathrm{PPV}=9.6 \%)$. High-risk patterns are more likely to be pathological when appearing at oxter and lower back but to be a physiological sign when appearing at Lower and upper of the prothorax. These high-risk patterns are significantly related to clinic sign. All these patterns are alsmost consistent during 6 hours after birth. Conclusions LUS is valid to differentiate healthy infants from potential patients who with mild respiratory difficulty. Four low-risk patterns have high value to predict healthy infants, but four high-risk patterns are not specific enough to discover patients. This criterion is valid for this 6 hours stage.
\end{abstract}

\section{Hosted file}

Main text.doc available at https://authorea.com/users/328608/articles/455798-the-ultrasoundimage-patterns-at-the-early-stage-after-birth-can-predict-health-neonates-a-nested-casecontrol-study

\section{Hosted file}

figure legend.docx available at https://authorea.com/users/328608/articles/455798-theultrasound-image-patterns-at-the-early-stage-after-birth-can-predict-health-neonates-anested-case-control-study 


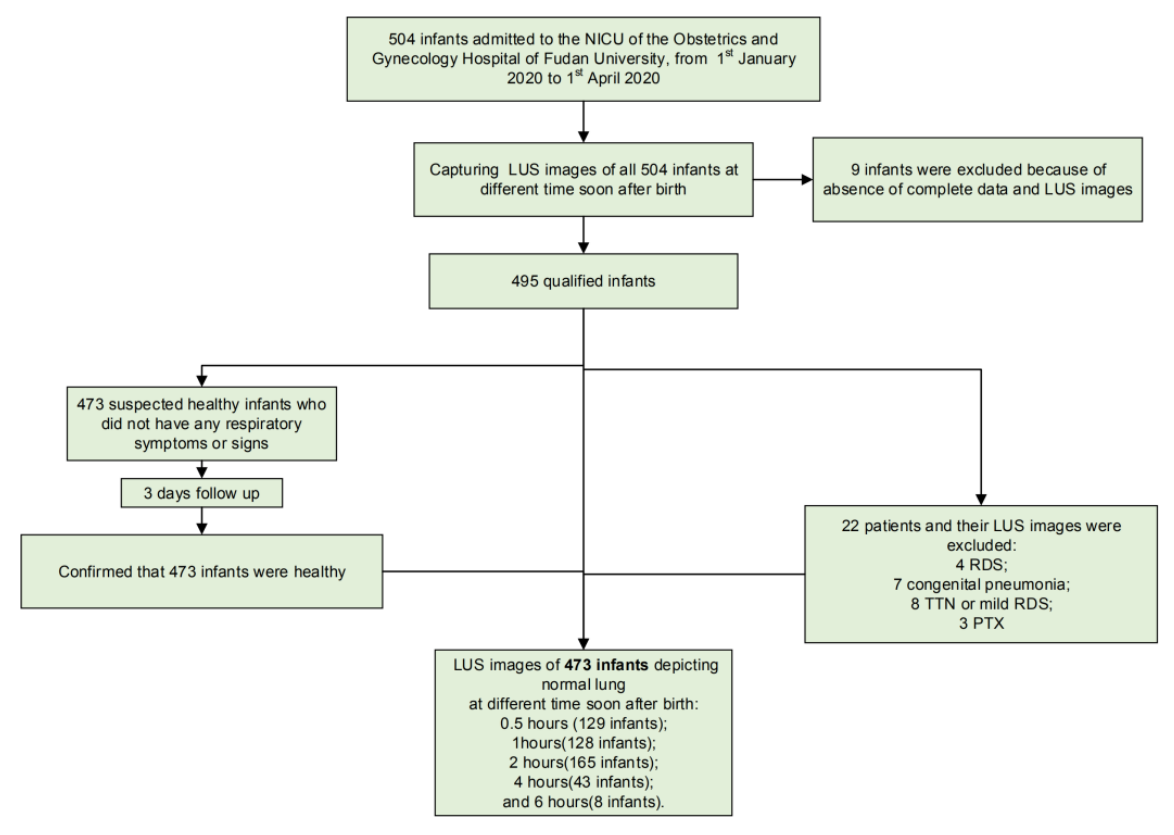

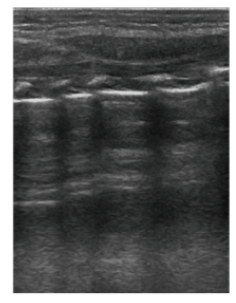

A

Pure A-line

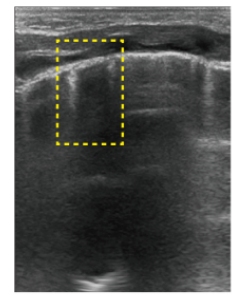

B

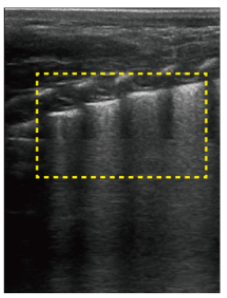

C

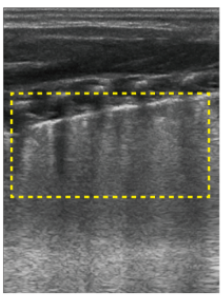

D

small amounts of DB moderate amounts of $\mathrm{DB}$ large amounts of $\mathrm{DB}$

\section{LOW-RISK}

\section{HIGH-RISK}

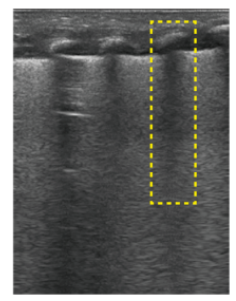

E

compact B-line

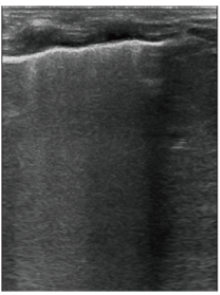

$\mathbf{F}$

Dense B-line

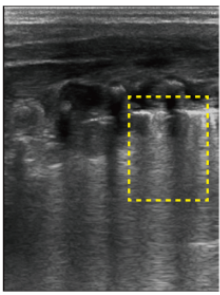

G

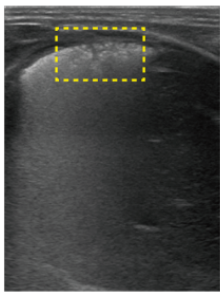

H

irregular consolidation mild consolidation with DB with air bronchograms 


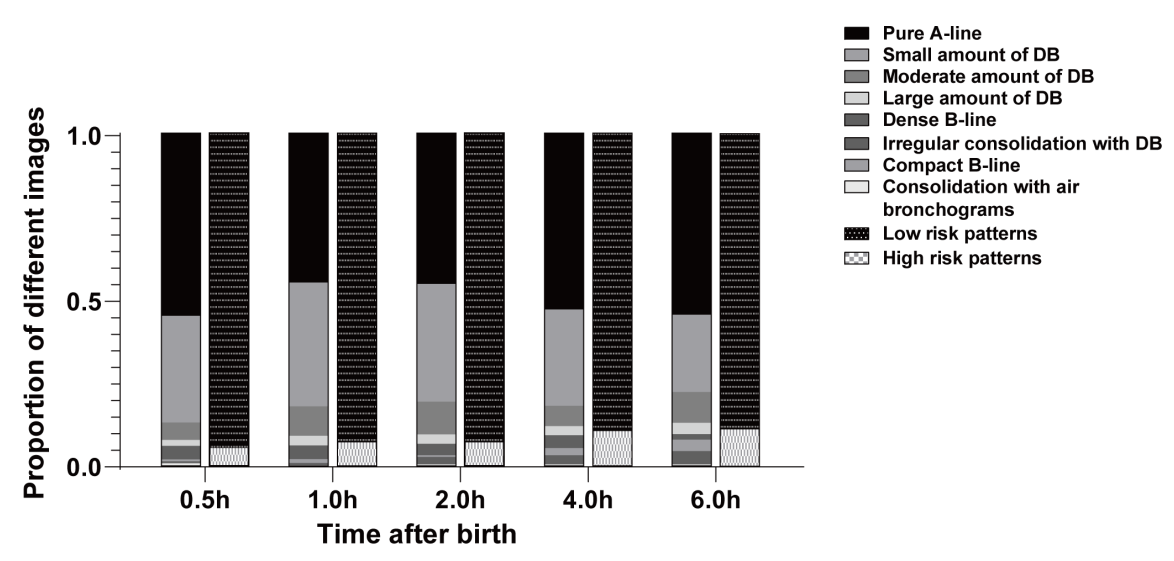

\section{Hosted file}

Table 1.doc available at https://authorea.com/users/328608/articles/455798-the-ultrasoundimage-patterns-at-the-early-stage-after-birth-can-predict-health-neonates-a-nested-casecontrol-study

\section{Hosted file}

Table 2.doc available at https://authorea.com/users/328608/articles/455798-the-ultrasoundimage-patterns-at-the-early-stage-after-birth-can-predict-health-neonates-a-nested-casecontrol-study 